\title{
ORAL FINDINGS IN CHILDREN WITH ATTENTION DEFICIT HYPERACTIVITY DISORDER AMONG A GROUP OF EGYPTIAN CHILDREN: A CROSS-SECTIONAL STUDY
}

Sara Gaber Hussein Galal", Randa Youssef Abd Al-Gawad*, Walaa Amin Fakher* ${ }^{* *}$, Dina Y. Affifi** and Rasha M. Hanafy Hatem*

\begin{abstract}
Objectives: To assess the oral findings of children with ADHD when compared to normal healthy children.

Materials and Methods: A sample of 78 children (age 4-12 years); 39 participants with ADHD and 39 participants without ADHD (control). Oral examination included the following parameters: caries, traumatic dental injuries (TDIs), malocclusions, dental wear, and enamel hypocalcification (EH). Additionally, oral hygiene and parafunctional oral habits were assessed by a questionnaire to parents/caregivers. Data were compared using Mann-Whitney U-tests and Chi-square tests.

Results: Children with ADHD have significantly lower values of deft/DMFT and frequencies of tooth brushing but higher values of parafunctional oral habits (bruxism, nail and object biting, tongue thrusting) and dental wear. No significant differences were found between children, with and without ADHD, in dmft, DMFT, TDIs, prevalence of malocclusion or EH.

Conclusion: Children with ADHD exhibited a poorer oral health behavior, higher risk for TDIs and malocclusion than non-ADHD children. Hence, there is a need for meticulous preventive dental care for them. Parents/caregivers need instructions for better supervision of oral hygiene to improve the poor oral health of children with or without ADHD.
\end{abstract}

\section{INTRODUCTION}

Attention-deficit/hyperactivity disorder is considered the most common childhood neurodevelopmental disorder that is characterized by inattention, hyperactivity, and impulsivity. These symptoms can last till adulthood in $65 \%$ of cases. ADHD has been reported to have a worldwide prevalence of $5.3 \%$ and it is more common in boys than in girls Ghanizadeh (2008); Serra-Negra et al. (2012) and Shooshtari et al. (2013).

* Pediatric Dentistry and Dental public Health Department, Faculty of Dentistry, Cairo University.

** Lecturer of Psychiatry, Faculty of Medicine, Cairo University 
Children with ADHD may present with additional medical issues, such as sleep disorders, encopresis, and enuresis, or it may be secondary to medical conditions, including traumatic brain injury, fetal alcohol exposure, lead intoxication, premature birth, epilepsy, and tic disorders Charles, (2010).

Attention-deficit/hyperactivity disorder has been extensively studied in the medical literature including neurology, psychiatry and psychology, but up to our knowledge, few studies have been published from the dental perspective. Since ADHD is a common disability, it is likely that dental practitioners will come across children, adolescents and even adults with this disorder in their practices. Accordingly, it is essential for the clinician to be familiar with this condition, the strategies for its management and its different effects on oral and dental health.

The symptoms of ADHD are generally treated through behavioral therapy, with or without pharmacological intervention. Medications of ADHD have been implicated in adverse oral effects, such as xerostomia and gingival enlargement, although evidence is scant. The current evidence is debatable regarding the severity and prevalence of dental caries, dental trauma, periodontal diseases, oral habits and tooth wear among patients with ADHD. Thus, the potential for increased risk of dental diseases in this population remains undetermined Chau et al. (2017b).

In the Arab world, few studies have been published on ADHD and these studies present conflicting results. Accordingly, in the Arab society and Egypt, there are limited data regarding the oral health of children with ADHD. Moreover, up to our knowledge, no published data was found about the oral and dental characteristics of children with ADHD in Egypt. Only single unpublished study carried out by Moawad et al. (2011) about oral findings, behavior and dental management of children with ADHD was found. Owing to the sparsity of these data, this study was conducted to evaluate different oral findings of children with ADHD in comparison to normal children.

\section{AIM OF THE STUDY}

The aim of this study was to detect the oral findings of children diagnosed with ADHD versus normal children.

\section{SUBJECTS AND METHODS}

\section{Study Design}

This is a cross-sectional analytical study.

\section{Sample Size Calculation}

- The sample size was calculated by a statistician expert using PASS version (15) based on the previous research by Atmetlla et al. (2005) entitled "Behavior and Orofacial Characteristics of Children with Attention-Deficit Hyperactivity Disorder during a Dental Visit"

- The sample size was calculated to be 78 children i.e. (39) cases per group: Group 1 (ADHD group) and Group 2 (Control/Non-ADHD group).

\section{Location}

- For Group 1: patients underwent oral and dental examination at the dental clinic located in the Center of Social and Preventive Medicine, Abo El-Resh children Hospital, Cairo, Egypt.

- For Group 2: patients underwent oral and dental examination at the Diagnosis Clinic of Pediatric Dentistry and Dental Public Health Department, Faculty of Dentistry, Cairo University.

\section{Subjects Selection}

\section{Eligibility criteria}

Children enrolled in the current study were selected according to the following inclusion and exclusion criteria: 


\section{Inclusion criteria}

\section{Group 1:}

- Children 4-12 years old, diagnosed with ADHD at the Child ADHD Clinic located in the Center of Social and Preventive Medicine, Abo ElResh children Hospital, Cairo University.

- Children with ADHD who were assessed using the K-SADS-PL.

- Both male and female patients were included.

\section{Group 2:}

- A matched group of apparently healthy children attending at the outpatient clinic of Pediatric Dentistry and Dental Public Health Department, Faculty of Dentistry, Cairo University.

\section{Exclusion criteria}

- Patients having other systemic or syndromic disorders, such as cerebral palsy or Down's syndrome.

- Patients complaining from psychiatric disorders other than ADHD such as autism, mental retardation or epilepsy.

\section{METHODS}

\section{Informed Consent}

\section{For both groups}

Interviews were held with the parents/caregivers of the participating children to explain the aim and all the details of the study and to obtain information regarding the child's personal data.

- The parents/caregivers were then asked to sign an informed consent, translated into Arabic, agreeing to clinical examination procedure and the use of the patient's data for scientific studies.

- The child also was asked for a verbal assent for clinical examination for only Group 2.

\section{Psychological Assessment for group 1}

- Patients and their parents were interviewed about the children's lifetime (present and past) DSM-V disorders $\boldsymbol{A P A}$, (2013) using the K-SADS-PL Kaufman et al. (1997). Briefly, $\mathrm{K}$-SADS-PL is a widely used semi-structured diagnostic interview designed to assess current and past episodes of psychopathology in children and adolescents according to DSM criteria. It consists of screens and supplemental diagnostic assessments for 20 psychiatric disorders (including ADHD).

\section{Oral and dental clinical examination}

- The principal researcher collected all patients' data and examined all the patients as well.

- A total 78 children participated in this study, consisting of 39 children, aged 4-12 years, diagnosed with ADHD and 39 healthy children who have neither any of the excluded psychiatric disorders nor systemic diseases.

- A custom-made chart was used to record the child's personal data, medical history, and dental history during the dental visit.

- All patients were examined on the dental chair, under operating light of the dental unit using were sterile gauze pads, cotton, and disposable diagnostic sets (dental mirrors, dental explorers and dental tweezers)

\section{Soft Tissue Examination}

- The intraoral soft tissue examination included checking lips and labial mucosa, buccal mucosa and vestibular mucosa, and gingiva alveolar mucosa and the tongue for the presence of oral lesions, abnormalities in color or presence of scars. Any abnormal data were recorded in the patient's assessment chart. Visual examination of the gum was performed and any abnormalities in color and consistency were recorded, 
as gingivitis (e.g.) rounded papilla, generalized or localized swelling, redness, ulceration or bleeding.

\section{Dental Examination:}

\section{Dental Caries:}

All teeth were examined for the presence/ absence of dental caries as follows:

- All teeth were cleaned of debris and plaque using sterile gauze and examined visually.

- If there were suspicious areas, then an explorer was used to check for the surface texture.

- Diagnosis of dental caries was carried out according to the World Health Organization (WHO, 1997) using (dmft), (DMFT) and (DMFT/deft) for primary dentition, permanent dentition, and mixed dentition respectively.

\section{Traumatic Dental Injuries (TDIs):}

- Teeth were thoroughly examined clinically for TDIs.

The presence/absence of dental trauma (cracks or coronal fractures) was reported by visual binary examination (YES/NO) respectively and the number of teeth affected were recorded.

\section{Occlusion:}

- Binary examination (YES/NO) was used for the presence/absence of malocclusion;

Teeth alignment was thoroughly examined (spacing, crowding, space loss, rotation). Bite was checked for (overbite and overjet).

\section{Developmental changes:}

- Found data of any abnormal findings in size (macro/microdontia), shape (fusion, germination, peg shaped, etc), number (hypo/hyerdontia), or structure (hypocalcification, amelogenesis/dentinogenesis imperfecta) were recorded in the patient's chart

\section{Oral habits}

- All data regarding different oral habits were obtained from verbal questionnaire answered by the parents in a face-to-face interview followed by clinical examination.

- Parents/caregivers were asked about history of oral habits (nail biting/lip biting/object biting/ digit sucking and bruxism)

- Special examinations were used for tongue thrusting (swallowing test) and mouth breathing (water holding test).

- Data was recorded by visual binary examination (YES/NO).

\section{Tooth brushing}

- Frequency of tooth brushing was obtained from verbal questionnaire answered by the parents/ caregivers and recorded in the patient's chart as: never, twice per day, once per day, or infrequent if the child did not brush his teeth on daily basis.

\section{Statistical analysis}

Statistical analysis was performed using a commercially available software program (SPSS 19; SPSS, Chicago, IL, USA). Qualitative values were presented as number and percentages. Chi square test was used to compare categorical data. Quantitative values were expressed as mean and standard deviation, and were compared between groups using independent $t$ test or Mann Whitney $\mathrm{U}$ test according to normality. Microsoft excel was used for generation of representative figures. The level of significance was set at $\boldsymbol{p}$-value $\leq 0.05$.

\section{RESULTS}

The present study was carried out to evaluate the different oral findings in 39 children with ADHD (Group 1), in comparison to 39 control group (Group 2) of apparently healthy non-ADHD children, all aged 4-12 years. The data was calculated as follows: 
In permanent dentition, (DMFT) score was recorded in 1 patient in Group 1 (DMFT=0), in comparison to 2 patients in Group 2, with mean (DMFT) score of $4 \pm 0$. Statistical analysis revealed that there was no significant difference between the two groups ( $\boldsymbol{p}$-value $>0.05$ ).

Regarding the prevalence of tooth wear, $30.8 \%$ in group 1 were found to have dental wear, in comparison to $12.6 \%$ of patients in group 2 . Statistical analysis of data revealed that the difference between the two groups was statistically significant ( $\boldsymbol{p}$-value $<0.05)$.

While regarding the number of hypocalcified teeth, in Group 1, each patient had 2 hypocalcified. In Group 2, three patients had one hypocalcified tooth and one patient had 4 hypocalcified teeth. The mean number of hypocalcified teeth was $0.21 \pm 0.61$ in Group 1, in comparison to $0.18 \pm 0.68$ in Group
2, with no significant difference between the two groups ( $\boldsymbol{p}$-value $>0.05$ ).

There was statistically significant between group 1 (ADHD) and group 2 (control) regarding nail biting, lip biting, bruxism, tongue thrusting and others while there was no statistically significant difference regarding digit sucking and mouth breathing.

In Group 1, (43.6\%) of patients reported infrequent tooth brushing, (43.6\%) reported that they never brushed their teeth, $(10.3 \%)$ reported brushing their teeth once per day, while (2.6\%) brushed their teeth twice daily. While in the Group $2,(48.7 \%)$ of patients reported infrequent brushing, $(17.9 \%)$ reported that they never brushed their teeth, (12.8\%) reported brushing their teeth once per day, while $(20.5 \%)$ brushed their teeth twice daily.

TABLE (1): The mean and SD of age in the two groups:

\begin{tabular}{ccc}
\hline Group & Mean \pm SD & $p$-value \\
\hline Group 1 (ADHD) & $8.40 \pm 1.92$ & 0.102 \\
\hline Group 2 (Control) & $7.68 \pm 1.91$ & \\
\hline
\end{tabular}

The statistical analysis was made using (Chi square test)

$P$-value $>0.05$ is considered insignificant

TABLE (2): Gender distribution among the study population in number(n) and percentage (\%):

\begin{tabular}{|c|c|c|c|c|c|}
\hline \multirow{2}{*}{ Gender } & \multicolumn{2}{|c|}{ Group 1 (ADHD) } & \multicolumn{2}{|c|}{ Group 2 (Control) } & \multirow{2}{*}{$p$-value } \\
\hline & Number (n) & Percentage (\%) & Number (n) & Percentage (\%) & \\
\hline Females & 7 & $17.9 \%$ & 18 & $46.2 \%$ & \multirow{2}{*}{$0.008^{*}$} \\
\hline Males & 32 & $82.1 \%$ & 21 & $53.8 \%$ & \\
\hline
\end{tabular}

The statistical analysis was made using (Chi square test)

*P-value $\leq 0.05$ is considered significant 
TABLE (3): Mean values of caries indices in the two groups:

\begin{tabular}{|c|c|c|c|c|}
\hline Dentition stage & Caries index & $\begin{array}{l}\text { Group } 1 \\
\text { (ADHD) }\end{array}$ & $\begin{array}{c}\text { Group } 2 \\
\text { (Control) }\end{array}$ & $p$-value \\
\hline \multirow{4}{*}{ Primary dentition (dmft) } & Mean (dt) & $6.67 \pm 7.64$ & $5.14 \pm 2.73$ & 0.909 \\
\hline & Mean (mt) & $0.00 \pm 0.00$ & $0.43 \pm 0.79$ & 0.329 \\
\hline & Mean $(\mathbf{f t})$ & $0.00 \pm 0.00$ & $1.71 \pm 1.25$ & $0.031^{*}$ \\
\hline & Mean (dmft) & $6.67 \pm 7.64$ & $7.29 \pm 3.4$ & 0.646 \\
\hline \multirow{8}{*}{ Mixed dentition (DMFT/deft) } & Mean (DT) & $0.45 \pm 0.98$ & $0.93 \pm 1.44$ & 0.129 \\
\hline & Mean (MT) & $0.00 \pm 0.00$ & $0.03 \pm 0.18$ & 0.260 \\
\hline & Mean (FT) & $0.00 \pm 0.00$ & $0.1 \pm 0.31$ & $0.048 *$ \\
\hline & Mean (DMFT) & $0.45 \pm 0.98$ & $1.03 \pm 1.43$ & $0.036^{*}$ \\
\hline & Mean (dt) & $1.82 \pm 2.32$ & $2.97 \pm 2.01$ & $0.009 *$ \\
\hline & Mean (et) & $1.05 \pm 1.21$ & $0.9 \pm 1.53$ & 0.403 \\
\hline & Mean (ft) & $0.00 \pm 0.00$ & $0.69 \pm 1.23$ & $0.00^{*}$ \\
\hline & Mean (deft) & $2.87 \pm 2.64$ & $4.4 \pm 2.5$ & $0.011 *$ \\
\hline \multirow{4}{*}{ Permanent dentition (DMFT) } & Mean (DT) & 0 & $2.50 \pm 2.12$ & 0.221 \\
\hline & Mean (MT) & 0 & $0.00 \pm 0.00$ & 1.00 \\
\hline & Mean (FT) & 0 & $1.50 \pm 2.12$ & 0.48 \\
\hline & Mean (DMFT) & 0 & $4 \pm 0$ & 0.157 \\
\hline
\end{tabular}

The statistical analysis was made using (Mann Whitney $U$ test)

*P-value $<0.05$ is considered a significant difference

$P$-value $>0.05$ is considered insignificant difference

TABLE (4): Prevalence of dental wear among the study groups in numbers (n) and percentages (\%):

\begin{tabular}{cccccc}
\hline \multirow{2}{*}{ Dental wear } & \multicolumn{2}{c}{ Group 1 (ADHD) } & \multicolumn{2}{c}{ Group 2 (Control) } & \multirow{2}{c}{-value } \\
\cline { 2 - 5 } & Number (n) & Percentage (\%) & Number (n) & Percentage (\%) & \\
\hline Yes & 27 & $30.8 \%$ & 1 & $2.6 \%$ & $0.001 *$ \\
\hline No & 12 & $84.6 \%$ & 38 & $97.4 \%$ & \\
\hline
\end{tabular}

The statistical analysis was made using (Chi square test) $* P$-value $<0.05$ is considered a significant difference

TABLE (5): Number of patients of enamel hypocalcification and mean number of hypocalcified teeth between the two groups:

\begin{tabular}{cccccc}
\hline Group & Number (n) & $\boldsymbol{p}$-value & Number of hypocalcified teeth & Mean \pm SD & $p$-value \\
\hline Group 1 (ADHD) & 4 patients & & 1 & 8 & $0.21 \pm 0.61$ \\
\cline { 1 - 4 } Group 2 (Control) & 4 patients & & 7 & $0.18 \pm 0.68$ & 0.86 \\
\hline
\end{tabular}

The statistical analysis was made using (chi square test) and (independent t test).

$P$-value $>0.05$ is considered insignificant difference, $S D=$ Standard deviation. 
TABLE (6): Prevalence of oral habits among the study groups in number(n) and percentage (\%):

\begin{tabular}{|c|c|c|c|c|c|}
\hline \multirow{2}{*}{ Oral habits } & \multicolumn{2}{|c|}{ Group 1 (ADHD) } & \multicolumn{2}{|c|}{ Group 2 (Control) } & \multirow{2}{*}{$p$-value } \\
\hline & Number (n) & Percentage (\%) & Number (n) & Percentage (\%) & \\
\hline Nail biting & 16 & $41.02 \%$ & 7 & $17.9 \%$ & $0.025 *$ \\
\hline Lip biting & 7 & $17.9 \%$ & 1 & $2.6 \%$ & $0.025 *$ \\
\hline Digit sucking & 6 & $15.4 \%$ & 2 & $5.1 \%$ & 0.135 \\
\hline Bruxism & 16 & $41.02 \%$ & 7 & $17.9 \%$ & $0.025 *$ \\
\hline Mouth breathing & 7 & $17.9 \%$ & 6 & $15.4 \%$ & 0.761 \\
\hline Tongue thrusting & 6 & $15.4 \%$ & 1 & $2.6 \%$ & $0.048^{*}$ \\
\hline Others & 16 & $41.02 \%$ & 2 & $5.1 \%$ & $0.00 *$ \\
\hline
\end{tabular}

The statistical analysis was made using (chi square test).

*P-value $<0.05$ is considered a significant difference. $P$-value $>0.05$ is considered insignificant difference.

Others (Pen biting, Plastic Biting, Clothes Biting, Paper Biting, Toes Nail Biting \& Wedging wood between teeth).

TABLE (7): Frequency of tooth brushing among the study groups in number (n) and percentage (\%):

\begin{tabular}{cccccc}
\hline \multirow{2}{*}{ Tooth brushing } & \multicolumn{2}{c}{ Group 1 (ADHD) } & \multicolumn{2}{c}{ Group 2 (Control) } & $\boldsymbol{p}$-value \\
\cline { 2 - 5 } & Number (n) & Percentage (\%) & Number (n) & Percentage (\%) & \multirow{2}{*}{$0.020 *$} \\
\hline Infrequent & 17 & $43.6 \%$ & 19 & $17.9 \%$ & $12.8 \%$ \\
\hline Never & 17 & $43.6 \%$ & 7 & $20.5 \%$ \\
\hline Once & 4 & $10.3 \%$ & 8 & 5 \\
\hline
\end{tabular}

The statistical analysis was made using (Chi square test)

*P-value $<0.05$ is considered a significant difference.

\section{DISCUSSION}

Attention deficit hyperactivity disorder is one of the most common chronic diseases in pediatrics which has been a topic of intense scientific research in the past two decades El-Bakry et al. (2019). It is considered as the most frequent neuropsychiatric disorder in school-age children with an increasing worldwide prevalence APA, (2013). ADHD is often confused with other neurological disorders; therefore, it is important for pediatric dentists to know how to identify and manage these children adequately with respect to their dental health Sujlana and Dang (2013).
This study was conducted to evaluate different oral findings in a group of children with ADHD compared to a control group of apparently healthy non-ADHD children. This helps to raise the awareness of pediatric dentists about the nature of the disease, its effect on oral and dental health, and the need to develop and implement specific strategies for the prevention and treatment of the associated oral diseases. Also it increases the awareness of parents and caregivers of ADHD children about the importance of seeking early dental examination and treatment. 
The results of the current study showed that the mean age of patients in the ADHD group was $8.40 \pm 1.92$ years, when the symptoms of ADHD are most evident, which was in accordance with $\boldsymbol{A P A}$, (2013). The mean age in the control group was $7.68 \pm 1.91$ with no significant difference in the age distribution among the study population.

In this study, boys $(82.15 \%)$ were more affected with ADHD than girls (17.9\%) with a ratio of (4.5:1) which is close to Grooms et al. (2006) with ratio of (4.4:1) and Ehlers et al. (2019) with ratio of (4:1) and lower than Chau et al. (2017a) with ratio of (6.75:1). Likewise, these results were in agreement with a previous Egyptian study by Aboul-ata and Amin (2018) who reported that ADHD was more prevalent among boys (33.8\%) than girls (6.8\%) with relatively a ratio of (4.9:1). Their crosssectional study was conducted on children, aged from 6-14 years, in Al Fayium city, Egypt.

To explain this ratio, it was suggested that girls with ADHD might be consistently under-identified and under-diagnosed. This was mostly explained by differences in the expression of the disorder among boys and girls. Females with ADHD were reported to have fewer hyperactive/impulsive symptoms and more inattentive symptoms when compared to males with ADHD. Furthermore, less disruptive behavior in females with ADHD might contribute to referral bias causing under-identification and lack of treatment for them Biederman et al., (2002).

Statistical analysis of dental caries prevalence in the current study showed that there is no statistically significant differences in the (DMFT) and (dmft) scores between the ADHD and non-ADHD groups in both permanent and primary dentition stages which goes in agreement with a study by PinarErdem et al. (2018) conducted a study on 58 ADHD children and 59 of the control group, all aged 6-15 years. Their results showed that no significant differences existed in DMFT/DMFS, and dmft/ $\mathrm{dmfs}$ values between the two groups.The lack of significant difference in the current study in the primary and secondary dentition stages could be due to the subgrouping of the participants into 3 groups which resulted in the presence of small number of patients of either fully permanent or fully primary teeth.

On the other hand, the findings of the current study were contrary to previous studies in which higher values of caries prevalence were presented in children with ADHD in the mixed dentition stage Grooms et al. (2006) and Chandra et al. (2009). Interestingly, some studies have reported significantly higher dental caries prevalence when incipient enamel caries was included and among older children with ADHD such as Blomqvist et al. (2011) and Kohlboeck et al. (2013).

Regarding dental trauma, the results of this study showed that the prevalence of TDIs was higher in the ADHD group (15.4\%) than in the control group $(7.7 \%)$, yet this difference has no statistical significance. Similar findings were reported by Chau et al. (2017a). The published literatures also suggested a higher prevalence of traumatic injuries in children with ADHD, possibly because of the nature of their functional impairments which may affect motor coordination problems, peer relationships, and increase the risk of physical abuse Silveira et al. (2020). Studies have reported trauma prevalence ranging from $2 \%$ to $42 \%$ in 1 - to 19-year-old children with ADHD, but few studies have reported statistically significant differences from children without ADHD Atmetlla et al. (2006) and Katz-Sagi et al. (2010). Generally, the existing studies concluded that ADHD may contribute to the adoption of risk behaviors and actions without considering the consequences, resulting in a greater predisposition to TDIs Uslu et al. (2007) and Perera et al. (2009).

Regarding dental wear, it was found to be higher in ADHD children than in the control group. Malki et al. (2005), as well as, Ertuğrul et al.(2018) also reported 
similar findings, but Chau et al., (2017a) found no significant differences in tooth wear between children with and without ADHD.Atmetlla et al., (2006) stated that the higher incidence of pathological attrition facets found in the ADHD group could be explained by more pronounced masticatory activity and a higher incidence of parafunctional habits as a means of releasing stress or anxiety which are common consequences of ADHD.

Regarding the prevalence of malocclusion, there was no differences between the two groups ( $\boldsymbol{p}$-value $=1)$. On the same note, Andersson and Sonnesen (2018) reported no significant differences in the occurrence of malocclusion and space anomalies between the ADHD and the control groups, which is also in agreement with Atmetlla et al., (2006). Generally, few studies have focused on orofacial characteristics of children with ADHD. Thus, it is still unclear if occlusion, space conditions or dental arch and palatal dimensions are affected in children with ADHD. Despite the fact that we need more data regarding the association between malocclusions and ADHD, projected general population rates enable us to estimate that a significant percentage of ADHD children may be affected by malocclusions due to the increased prevalence of parafunctional habits Atmetlla et al., (2006).

Upon examination of the teeth, number of patients with EH was analyzed with no difference between ADHD group and control group (p-value $=1$ ), while number of teeth affected were higher in the ADHD group with no significant difference. Unlike Atmetlla et al., (2006) who reported that a higher percentage of $\mathrm{EH}$ was seen in the ADHD group (5.4 teeth) in contrast to the control group (3.6 teeth), with a statistically significant difference $(\boldsymbol{p}$-value $=0.05)$. They stated that the higher occurrence of enamel opacities found in the ADHD group could be attributed to the embryonic origin of its structure just as the ectoderm of the central nervous system can suffer injury at the same time of development.

Regarding the incidence of oral habits, it was found that ADHD children showed higher percentages than the control group with a statistically significant difference. The highest incidences were reported in nail biting, bruxism, tongue thrusting and other miscellaneous habits (Pen biting, Plastic Biting, Clothes Biting, Paper Biting, Toes Nail Biting \& Wedging wood between teeth). No statistical significant difference was found between the two groups as regards of mouth breathing and digit sucking.

Studies on the prevalence of bruxism suggested that its prevalence was higher in children with ADHD than in those without ADHD. Another study concluded that ADHD signs had a significant effect on sleep bruxism in school-age children. Similarly, Chau et al.,(2017a) found that children with ADHD had a significantly higher frequency of parent-reported bruxism than did children without ADHD. On the contrary, Ehlers et al., (2019) found that the presence of bruxism was similar in both ADHD and control groups.

Regarding soft tissue examination, only presence or absence of gingivitis was recorded, but clinical examinations such as assessment of GBI could not be conducted due to low compliance and short attention span of the ADHD participants. However, Aminabadi et al. (2016) found that there was no statistically significant differences in GBI between ADHD and non-ADHD participants, whereas in other studies, statistically significant higher gingival bleeding was found in children with ADHD Blomqvist et al. (2011) and Chau et al. (2017a).

Concerning oral hygiene practice, there was a statistically significant difference in the frequency of tooth brushing between the two groups ( $\boldsymbol{p}$-value= $0.020)$. ADHD children showed a higher percentage of patients who never brushed their teeth (43.6\%) versus (17.9\%) in the control group. On the other hand, non-ADHD children showed higher percentage of children who brush their teeth once 
and twice daily with percentages of $(12.8 \%)$ and $(20.2 \%)$ respectively versus $(10.3 \%)$ and $(2.6 \%)$ in the ADHD group. Chandra et al., (2009) reported similar findings when the frequency of brushing was compared between the ADHD and non-ADHD groups. They found that (15.9\%) ADHD subjects do not brush their teeth everyday compared to nonADHD patients who brush their teeth every day. This finding was also in agreement with the study done by Blomqvist et al. (2007). However, other studies reported no difference between children with and without ADHD regarding tooth brushing habit Ehlers et al. (2019).

The findings of the present study could be explained by the executive function deficits that ADHD children suffered from and may manifest as forgetfulness, trouble concentration and difficulty in planning and co-ordinating everyday routine monotonous tasks such as tooth brushing Tarver et al. (2014). In addition, parent-child conflict, which often occurs in families that raise children with ADHD, may also have an impact on health behavior in the field of oral hygiene; a persistent, purposeful neglect of brushing one's teeth can be a sign of defiance and resisting authority which are features of children with ADHD Blomqvist et al. (2007).

\section{CONCLUSIONS}

- Children with ADHD may constitute a risk group for dental caries.

- Children with ADHD are at higher risk of oral and dental trauma.

- Parafunctional oral habits are more frequent in children with ADHD which may lead to different oral and dental implications such as dental wear, muscle fatigue, localized gingivitis or periodontitis and increase the risk of malocclusions.

- Children with ADHD have poorer oral health behavior in the terms of frequency of tooth brushing.

\section{RECOMMENDATIONS}

- Early caries preventive measures such as fluoride treatments, fissure sealants, oral hygiene and dietary advice and regular dental check-ups are recommended for both children with and without ADHD. Children with ADHD should be followed up at shorter intervals between dental examinations to prevent caries progression.

- As ADHD children are of higher risk of dental trauma, instruction of parents/caregivers on appropriate protocol following dental trauma is mandatory, as well as, reviewing safety issues suitable to the age of the child, such as mouth guards to prevent oral-facial trauma.

- Regular check-up for children with parafunctional oral habits for early detection of malocclusion and consequently, early orthodontic intervention if needed. Night guard is recommended in cases of bruxism to reduce the incidence of dental wear, temporomandibular joint disorders and muscle fatigue.

- Tooth brushing charts for ADHD children to take home and mark off daily are more recommended than verbal instruction in order to overcome their forgetfulness and low concentration.

- Parents/caregivers of children with ADHD must receive good oral and dental educational programs to be aware of the condition of their children's oral health.

- Gap of knowledge between child psychiatrists and pediatric dentists should be filled so that collaboration can serve to the improvement of children with developmental disorders.

- More researches on oral health and ADHD are required, particularly in larger scale.

\section{REFERENCES}

- Aboul-ata, M.A. and Amin, F.A. (2018): The Prevalence of ADHD in Fayoum City (Egypt) Among School-Age Children: Depending on a DSM-5-Based Rating Scale. J Atten Disord., 22(2):127-33. 
- American Psychiatric Association (APA). (2013): Diagnostic and Statistical Manual of Mental Disorders 5th ed., p. 59-65, American Psychiatric Association. Arlington, VA

- $\quad$ Aminabadi, N.A., Najafpour, E., Erfanparast, L., Jamali,Z., Pournaghi-Azar, F., Tamjid-Shabestari, S., and Shirazi, S. (2016): Oral Health Status, Dental Anxiety, and Behaviormanagement Problems in Children with Oppositional Defiant Disorder. Eur J Oral Sci., 124(1):45-51.

- Andersson, H., and Sonnesen, L. (2018): Sleepiness, Occlusion, Dental Arch and Palatal Dimensions in Children Attention Deficit Hyperactivity Disorder (ADHD). Eur Arch Paediatr Dent., 19(2):91-97.

- Atmetlla, G., Burgos, V., Carrillo, A., and Chaskel, A. (2006): Behavior and Orofacial Characteristics of Children with Attention-Deficit Hyperactivity Disorder during a Dental Visit. J Clin Pediatr Dent., 30(3):183-90.

- Biederman, J., Mick, E., Faraone, S. V., Braaten, E., Doyle, A., Spencer, T., et al. (2002): Influence of gender on attention deficit hyperactivity disorder in children referred to a psychiatric clinic. Am. J. Psychiatry., 159(1): 36-42.

- Blomqvist, M., Holmberg, K., Fernell, E., Ek, U., and Dahllöf, G. (2007): Dental Caries and Oral Health Behavior in Children with Attention Deficit Hyperactivity Disorder. Eur J Oral Sci. , 115(3):186-191.

- Blomqvist, M., S. Ahadi, E. Fernell, Ulla Ek, and G. Dahllöf. (2011): Dental Caries in Adolescents with Attention Deficit Hyperactivity Disorder: A Populationbased Follow-up Study. Eur J Oral Sci., 119(5):381-385.

- Chandra, P, Anandakrishna, L., and Ray, P. (2009): Caries Experience and Oral Hygiene Status of Children Suffering from Attention Deficit Hyperactivity Disorder. J Clin Pediatr Dent., 34(1):25-29.

- Charles, J. M. (2010): Dental care in children with developmental disabilities: attention deficit disorder, intellectual disabilities, and autism. J Dent Child., 77(2): 84-91.

- Chau, Y. C., Lai, K. Y., McGrath, C. P., and Yiu, C. K. (2017a): Oral health of children with attention deficit hyperactivity disorder. Eur J Oral Sci., 125(1): 49-54.

- Chau, Y. C., Peng, S.M., McGrath, C. P., and Yiu, C. K. (2017b): Oral health of children with attention deficit hyperactivity disorder: Systematic review and metaanalysis. J Atten Disord., 24(7): 947-962.
- Ehlers, V., Callaway, A., Wantzen, S., Patyna, M., Deschner, J., and Azrak B. (2019): Oral Health of Children and Adolescents with or without Attention Deficit Hyperactivity Disorder (ADHD) Living in Residential Care in Rural Rhineland-Palatinate, Germany. BMC Oral Health. 19(1): 1-10.

- $\quad$ El-Bakry, A., El Safty, A. M., Abdou, A. A., Amin, O. R., Ayoub, D. R., and Afifi, D. Y. (2019): Zinc Deficiency in Children with Attention-Deficit Hyperactivity Disorder. Egypt J Psychiatry., 40(2): 95-103.

- $\quad$ Ertuğrul, C. Ç., Kırzıoğlu, Z., Aktepe, E., and Savaş, H. B. (2018): The effects of psychostimulants on oral health and saliva in children with attention deficit hyperactivity disorder: A case-control study. Niger J Clin Pract, 21(9)., 21(9): 1213-1220.

- Ghanizadeh, A. (2008): Association of nail biting and psychiatric disorders in children and their parents in a psychiatrically referred sample of children. Child and Adolesc Psychiatry Ment Health., 2(1): 2-13.

- Grooms, T., Michael, M. A., Roberts, M., and McIver, F.T. (2006): Caries Experience Associated with AttentionDeficit/Hyperactivity Disorder. J Clin Pediatr Dent., 30(1): 3-8.

- Katz-Sagi, H., Redlich, M. T., Brinsky-Rapoport, I. M., and. Ram, D. (2010): Increased Dental Trauma in Children with Attention Deficit Hyperactivity Disorder Treated with Methylphenidate-a Pilot Study. J Clin Pediatr Dent., 34(4): 287-90.

- Kaufman, J., Birmaher, B., Brent, D., Rao, U. M., Flynn, C., Moreci, P., and Ryan, N. (1997): Schedule for affective disorders and schizophrenia for school-age childrenpresent and lifetime version (K-SADS-PL): initial reliability and validity data. J Am Acad Child Adolesc Psychiatry., 36(7): 980-988.

- Kohlboeck, G., Heitmueller, D., Neumann, C., Tiesler, C., Heinrich, J., Heinrich- Weltzien, R., and Kühnisch, J. (2013): Is there a relationship between hyperactivity/ inattention symptoms and poor oral health? Results from the GINIplus and LISAplus study. Clin Oral Investig., 17(5): 1329-1338.

- Malki, G., Zawawi, K., Melis, M., and Hughes, C. (2005): Prevalence of bruxism in children receiving treatment for attention deficit hyperactivity disorder: a pilot study. J Clin Pediatr Dent., 29(1): 63-67. 
- $\quad$ Moawad, R., Sayed, M., Ezzat, A. (2011): Oral findings, behavior and dental management of children with attention deficit hyperactivity disprder. Thesis (M.Sc.), Cairo University, Faculty of Dentistry, Department of Pediatric Dentistry and Dental public Health.

- Perera, H., Fernando, S. M., Yasawardena, A. D., \& Karunaratne, I. (2009): Prevalence of Attention Deficit Hyperactivity Disorder (ADHD) in Children Presenting with Self-Inserted Nasal and Aural Foreign Bodies. Int J Pediatr Otorhinolaryngol., 73(10):1362-1364.

- $\quad$ Pinar-Erdem, A., S., Kuru, E. S., Ürkmez, E., Sepet, H., Günes, N., Yildiz, N.T., and Külekçi, G. (2018): Oral Health Status and Its Relation with Medication and Dental Fear in Children with Attention-Deficit Hyperactivity Disorder. Niger J Clin Pract., 21(9):1132-1338.

- $\quad$ Serra-Negra, J. M., Paiva, S. M., Auad, S. M., RamosJorge, M. L., and Pordeus, I. A. (2012): Signs, symptoms, parafunctions and associated factors of parent-reported sleep bruxism in children: a case-control study. Braz dent J., 23(6): 746-752.

- Shooshtari, H., Bidaki, R., Fili, J., and Zarafshan, H.
(2013): Sleep disturbances in children with ADHD: a study conducted at Hazrat Rasoul Akram Hospital, Tehran. Sri Lanka J Psychiatry., 4(1): 14-17.

- Silveira, A. L., Magno, M. B., and Soares, T. R. (2020): The Relationship between Special Needs and Dental Trauma. A Systematic Review and Meta-Analysis. Dent Traumatol., 36(3): 218-236.

- Sujlana, A., and Dang, R. (2013): Dental care for children with attention deficit hyperactivity disorder. J Dent Child., 80(2): 67-70.

- $\quad$ Tarver, J., David D., and Kapil S. (2014): Attention-deficit Hyperactivity Disorder (ADHD): An Updated Review of the Essential Facts. Child Care Health Dev., 40(6):762-774.

- Uslu, M., Uslu, R., Eksioglu, F., and Ozen, N. E. (2007): Children with Fractures Show Higher Levels of ImpulsiveHyperactive Behavior. Clin Orthop Relat Res., 460:192-195.

- World Health Organization -WHO. (1997): Oral health surveys: basic methods, $4^{\text {th }}$ ed., p. 41-42, Geneva. www. paho.org/hq/dmdocuments/2009/OH_st_Esurv.pdf. Accessed June 4, 2020. 